\title{
Las tecnologías digitales en contextos interculturales
}

\section{Digital technologies in intercultural contexts}

Pedro Hepp K. ${ }^{\otimes}$

\begin{abstract}
RESUMEN
El presente trabajo ofrece un panorama general sobre la educación chilena con énfasis en los sectores de población más vulnerable. En seguida, aborda el uso de las tecnologías digitales en contextos interculturales en general y en el ámbito mapuche en particular. A continuación describe el estado de las tecnologías digitales en Chile y las oportunidades que esta situación ofrece para el trabajo educativo intercultural. Finalmente, presenta la línea de tecnologías digitales en contextos indígenas e interculturales del Núcleo Iniciativa Científica Milenio Centro de Investigación en Educación en Contexto Indígena e Intercultural (CIECII) de la Universidad Católica de Temuco.
\end{abstract}

PALABRAS CLAVE: TIC, interculturalidad, educación, vulnerabilidad

\section{ABSTRACT}

The present work offers a general view about Chilean education with an emphasis on the most vulnerable sectors of population. It also covers the use of digital technologies in intercultural contexts in general and in the mapuche field in particular. It also describes the state of the digital technologies in Chile and the opportunities that this situation offers for the intercultural educational work. Finally, it presents the line of the digital technologies in indigenous and intercultural context of the Núcleo Iniciativa Científica Milenio Centro de Investigación en Educación en Contexto Indígena e Intercultural (CIECII) of the Universidad Católica de Temuco.

KEY WORDS: TIC, intercultural education, vulnerability 


\section{La realidad educativa chilena en contextos de pobreza}

De acuerdo a mediciones internacionales de la calidad de los aprendizaje (TIMSS ${ }^{1}$ y PISA $^{2}$ ), nuestro país se ubica levemente sobre el promedio de Latinoamérica en cuanto a los resultados en lenguaje, matemática y ciencias, pero está muy por debajo del promedio de los países desarrollados. En el gráfico 1 se pueden apreciar los resultados obtenidos por nuestro país en la evaluación PISA desarrollada por la OECD.

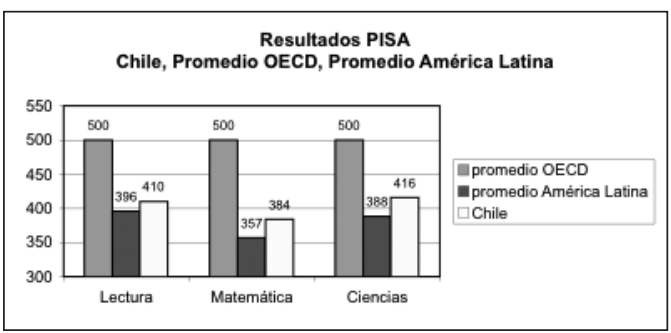

Gráfico 1. Resultados de PISA

En PISA se constató que en Chile, en el área de lectura, los estudiantes evaluados obtuvieron 90 puntos menos que el promedio de los países de la OECD y están muy lejos de los 546 puntos que obtuvieron los estudiantes finlandeses, los mejor ubicados en el ranking internacional. Al comparar los puntajes de estudiantes chilenos del $25 \%$ de mejor rendimiento, se observó que estos tienen un desempeño equivalente al $25 \%$ de los alumnos con más bajo rendimiento de Canadá y Japón, o equivalente a los alumnos promedio de Portugal y Grecia. Los mejores estudiantes de nuestro país tienen un desempeño igual a los peores estudiantes canadienses o japoneses.

Al analizar la calidad de la educación en las escuelas chilenas, la prueba SIMCE $^{3}$ refleja grandes diferencias en los niveles de aprendizaje entre las escuelas particulares pagadas y las escuelas subvencionadas, donde se concentra más del $90 \%$ de la matrícula escolar. Numerosas escuelas rurales y urbanomarginales tienen rendimientos inaceptablemente bajos, con niveles de aprendizaje en lectura-escritura, matemáticas y ciencias que revelan una gran precariedad educativa, una deficitaria gestión de la dirección, profesores con insuficiente formación pedagógica y una comunidad que se involucra escasamente en los aprendizajes de sus niños.

\section{La educación en la Región de La Araucanía}

De acuerdo a los datos de la prueba SIMCE que realiza anualmente el Ministerio de Educación para la $4^{\circ}$ año básicos, la Región de La Araucanía, que cuenta con 1.341 escuelas, de las cuales el $67 \%$ son rurales, ha obtenido históricamente entre los resultados más bajos del país en Matemática, Lenguaje y Ciencias.

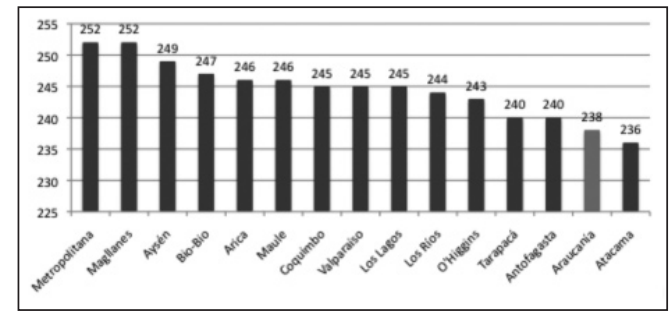

Gráfico 2. Resultados SIMCE 2008 en Matemática por región

En el SIMCE 2008 la Región de La Araucanía avanzó y abandonó por primera vez el último lugar en Matemática y ya está por sobre otras tres regiones en Lenguaje.

2 TIMSS: Trends in International Masthematics and Science Study: http://nces.ed.gov/timss/index.asp

3 PISA: OECD Programme for International Student Assessment: http://www.pisa.oecd.org/

4 SIMCE: Sistema nacional de medición de resultados de aprendizaje del Ministerio de Educación de Chile: http://www.simce. $\mathrm{cl} /$ 
Sin embargo, los resultados regionales esconden la realidad de las escuelas en las comunas más pobres y con mayor proporción de estudiantes mapuche, donde los resultados son significativamente inferiores al promedio regional (ver por ejemplo http: //www.visua(iza.cl), como se muestra en el siguiente gráfico:

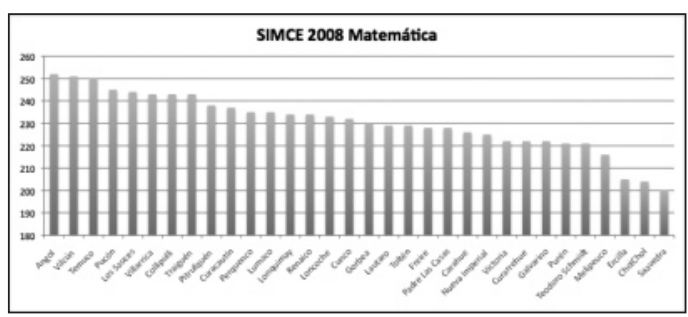

Gráfico 3. Resultados SIMCE 2008 en Matemática por comuna

Cabe destacar que las comunas de mayor pobreza (según índice de vulnerabilidad de encuesta CASEN $^{4}$ ) tienen en general, peores resultados de aprendizaje que las de menor vulnerabilidad.

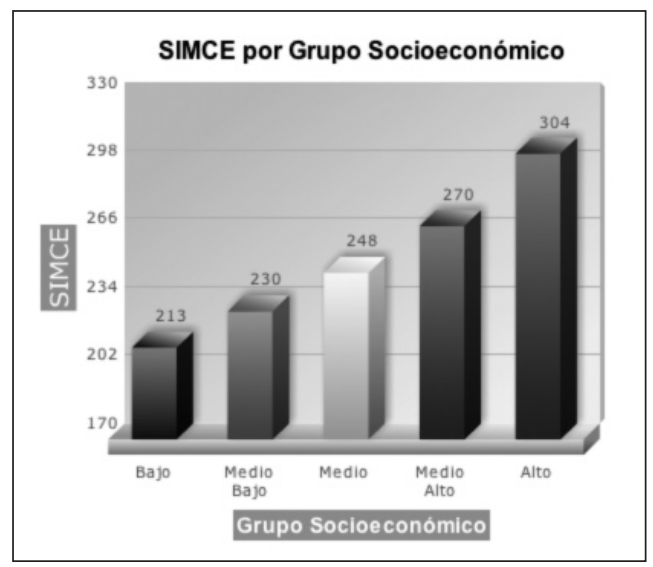

Junto a la mayor pobreza, es en estas comunas donde se concentra la mayor proporción de población mapuche, lo que ha llevado al error de asociar causalidad de pobreza de resultados educativos con factores étnicos.

\section{Las tecnologías digitales en la educación chilena}

La experiencia nacional e internacional de uso de tecnologías digitales en educación indica que la clave para lograr un uso efectivo radica en la actitud de cada profesor y de la dirección del establecimiento por usar bien estos recursos [Barak, 2006; Loveless, 2003; Pelgrum, 2001 y 2002]. Las tecnologías digitales pueden mejorar la calidad de los procesos de gestión que requieren las escuelas, desde el profesor de aula hasta la dirección del establecimiento. Por otra parte, los jóvenes que tienen acceso frecuente a estas tecnologías han demostrado que pueden usarlas de maneras creativas, acorde a sus intereses y con mayor flexibilidad que los adultos. Ejemplos de esto son la creación de música digital, las comunicaciones (chats, blogs), juegos, producciones multimediales (clips de video, presentaciones, web), programación y construcción de artefactos como robots de bajo costo, creación de objetos físicos a partir del diseño digital (FABs), etc.

El desafío es unir ambos mundos: los objetivos educativos con la buena predisposición de los jóvenes hacia estas tecnologías. La experiencia de muchas escuelas adscritas al Programa "Partners in Learning 5 " de Microsoft, activo en más de 100 países, y al propio Programa Enlaces del Ministerio de Educación de Chile ${ }^{6}$, dan cuenta de las múltiples posibilidades educativas y creativas que tiene esta unión.

La literatura internacional sugiere que una buena dosis de tecnologías digitales para el apoyo de los procesos de gestión, más una adecuada preparación de los profesores para el uso de tecnologías digitales en aula y un acceso frecuente de los jóvenes a las tecnologías a partir de sus intereses, son tres elementos que pueden agregarle gran valor a la escuela como espacio educativo [Zhao 2002].

El Programa Enlaces [Hepp, 2003; Hepp, 2009; Hinostroza, 2009], de casi dos década de antigüedad, ha permitido a los estudiantes

4 CASEN: Encuesta de Caracterización Socioeconómica Nacional. http://www.mideplan.cl/casen/descripcion.html

5 Partners in Learning: http://www.microsoft.com/education/pil/partnersinlearning.aspx

6 Enlaces: Programa de Informática Educativa del Ministerio de Educación de Chile. http://www.enlaces.cl/ 
de escuelas chilenas el acceso a las tecnologías digitales. El siguiente cuadro muestra las cifras de Enlaces del año 2008 y la proyección al 2012:

\begin{tabular}{|l|c|c|}
\hline \multicolumn{1}{|c|}{ ENLACES } & 2008 & 2012 \\
\hline Alumnos por PC & 25 & 10 \\
\hline \% Matricula con acceso a TIC & $95 \%$ & $100 \%$ \\
\hline Escuelas con Internet & $75 \%$ & $100 \%$ \\
\hline Aulas con TIC & 3.000 & 10.000 \\
\hline PC para alumnos & 20.000 & $¿ ?$ \\
\hline
\end{tabular}

\section{Contenidos digitales interculturales}

El tema intercultural está ampliamente incorporado en Internet, desde múltiples perspectivas, formatos e idiomas. Una búsqueda en Internet realizada en mayo del año 2009, arroja las siguientes respuestas:

\begin{tabular}{|l|c|c|c|}
\hline PALABRA O FRASE BUSCADA & $\begin{array}{c}\text { Nro. de respues- } \\
\text { tas en Google } \\
\text { (Sitios) }\end{array}$ & $\begin{array}{c}\text { Nro. de respues- } \\
\text { tas en Youtube } \\
\text { (Vídeos) }\end{array}$ & $\begin{array}{c}\text { Nro. de respuestas } \\
\text { en Flickr (Fotos) }\end{array}$ \\
\hline intercultural & 8.320 .000 & 3.760 & 7.075 \\
\hline mapuche & 1.810 .000 & 2.410 & 7.295 \\
\hline aborígenes (español) & 1.290 .000 & 1.550 & 1.338 \\
\hline aborigines (inglés) & 4.060 .000 & 10.600 & 7.382 \\
\hline mapuche y tecnologías digitales & 29.700 & 164 & 257 \\
\hline digital technologies and aborigines & 178.000 & & \\
\hline mapudungun & 516.000 & 164 & \\
\hline
\end{tabular}

Entre los sitios Internet que incorporan temas interculturales en español y tecnologías digitales, cabe destacar LOF Digital: http:// www.lofdigital.org.ar/ y Aula Intercultural: http://www.aulaintercultural.org, ambos describiendo un proyecto de capacitación en el uso de las tecnologías de información y comunicación para jóvenes indígenas.

En las referencias se entrega una lista de sitios Internet internacionales, con contenidos interculturales y uso de tecnologías digitales.
En algunos de estos sitios se aborda el uso de tecnologías digitales por parte de los pueblos originarios, en un sentido amplio. Por ejemplo, entre los aportes de la tecnología digital, mirada desde la perspectiva aborigen, se cuentan:

1. Mayor capital social: redes (Las TIC como unificadoras - foros, portales): redes de apoyo y de información entre comunidades, fortalecimiento político y social de comunidades, difusión de derechos, registro digital de la cultura, la lengua, tradiciones, música, testimonios de ancianos. 
2. Mejor educación: acceso a escuelas virtuales, a fuentes de información, a recursos didácticos en línea.

3. Mejor salud: Telemedicina (Canadá).

4. Mejor economía: redes de comercialización, difusión, acceso a créditos, beneficios, información de mercado, clientes, asociatividad.

Y entre las amenazas de las tecnologías digitales a las comunidades indígenas, el siguiente es un resumen obtenido de diversas páginas de Internet:

1. Aumentos de las desigualdades: acceso limitado, capacitación descontextualizada de la cultura local.

2. Homogeneización e imposición: los contenidos, lengua o cultura dominantes en Internet pueden tener efectos negativos al uniformar ideas, preferencias y visiones del mundo. Así, se podrían reproducir las relaciones de dominación existentes en la sociedad.

3. Aislamiento y fragmentación del mundo real: las TIC pueden provocar distanciamiento y aislamiento si el mundo virtual recibe más atención que el mundo real.

Ejemplos encontrados en Internet que ilustran la perspectiva de algunas comunidades aborígenes sobre las tecnologías digitales:

[Aboriginal Voice - Alberta, Canadá]:

"... Creo que todos estarán de acuerdo en que la comunidad aborigen está fragmentada. Competimos entre nosotros por recursos y discutimos al interior de nuestras organizaciones sobre una variedad de temas políticos, económicos y de derechos de herencia.

Atados como estamos a nuestra cultura y tradiciones, sentimos sin embargo la fuerte necesidad de medios materiales para la supervivencia económica en el mundo moderno.
Veo que las TIC ofrecen una ruta para superar estos conflictos $y$, si bien no resolverán las tensiones internas, al menos le darán un sentido constructivo en la comunidad amplia. Las TIC tienen el potencial de agregar coherencia a nuestros propios debates internos y empoderarnos para trabajar juntos. Una mayor comunicación puede generar foros en los que todos nuestros temas puedan ser ventilados y discutidos abiertamente. Puede también lograr una mejor articulación de lo que nosotros, como personas y como grupo minoritario en Canadá, queremos de nuestros líderes políticos y de nosotros mismos".

Internet también constituye una herramienta positiva para los pueblos indígenas de América Latina. Los siguientes son algunos testimonios extraídos de diversos sitios en Internet, expresados desde la perspectiva de grupos o comunidades aborigenes:

"... Internet ha permitido la posibilidad de comunicarse salvando el aislamiento geográfico en el que habitualmente viven las poblaciones indígenas...

... Internet ha permitido que las propias comunidades indígenas puedan entrar en contacto entre ellas, colaborando entre sí para apoyar sus esfuerzos de desarrollo, fortalecimiento organizativo, autogestión y afirmación cultural...

... también se ha facilitado el contacto directo con organizaciones regionales y nacionales, agencias de cooperación...

... las nuevas tecnologías han empezado a ser empleadas (bajo lo que se conoce como comercio electrónico) por parte de las poblaciones indígenas para ofrecer sus productos en todo el mundo. Internet permite a estas poblaciones tratar directamente con los consumidores, lo que le supone mayores ingresos en la medida que desaparece el papel de los intermediarios...

En Chile pueden revisarse dos iniciativas importantes con contenido mapuche educativo de acceso masivo. En ambos casos hay un esfuerzo por incorporar mayoritariamente contenidos históricos y culturales y asociarlos con el currículo oficial de modo que puedan ser aprovechados en aula. 
En primer lugar están los contenidos del Portal Educarchile ${ }^{7}$ cuyo buscador ofrece 582 recursos educativos organizados según se muestra en la siguiente figura:

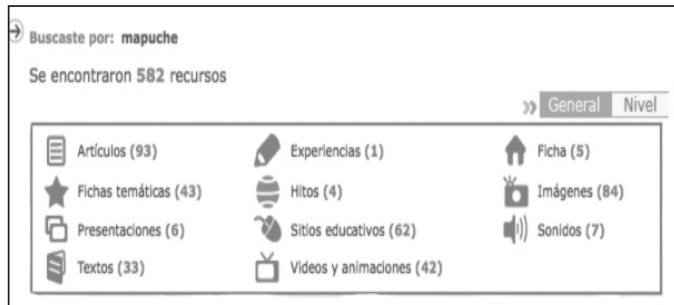

Resultados de búsqueda por "mapuche" en el Portal Educarchile.

En cuanto al Programa Enlaces, este ha llevado a cabo diversas iniciativas en el ámbito intercultural, desde apoyar la traducción del sistema operativo Windows de Microsoft al mapudungun, hasta desarrollar CDs con contenidos mapuche.

Un ejemplo destacable de contenidos en CD es el software "Kimkantuain Aprender Haciendo" construido por el Instituto de Informática Educativa de la Universidad de La Frontera ${ }^{8}$ con el apoyo del Instituto de Estudios Indígenas de la misma casa de estudios. Este $\mathrm{CD}$, orientado a educación básica, con guías para profesores y actividades para alumnos, busca que los alumnos aprendan acerca de la cultura mapuche, su idioma (contiene un grafemario y diccionario multimedial mapuche-español), la relación con el medio ambiente en la cultura mapuche, temas del hogar mapuche (con relatos hablados $\mathrm{y}$ animados) y herramientas interactivas para pintar. A continuación se muestran algunas imágenes de este software:
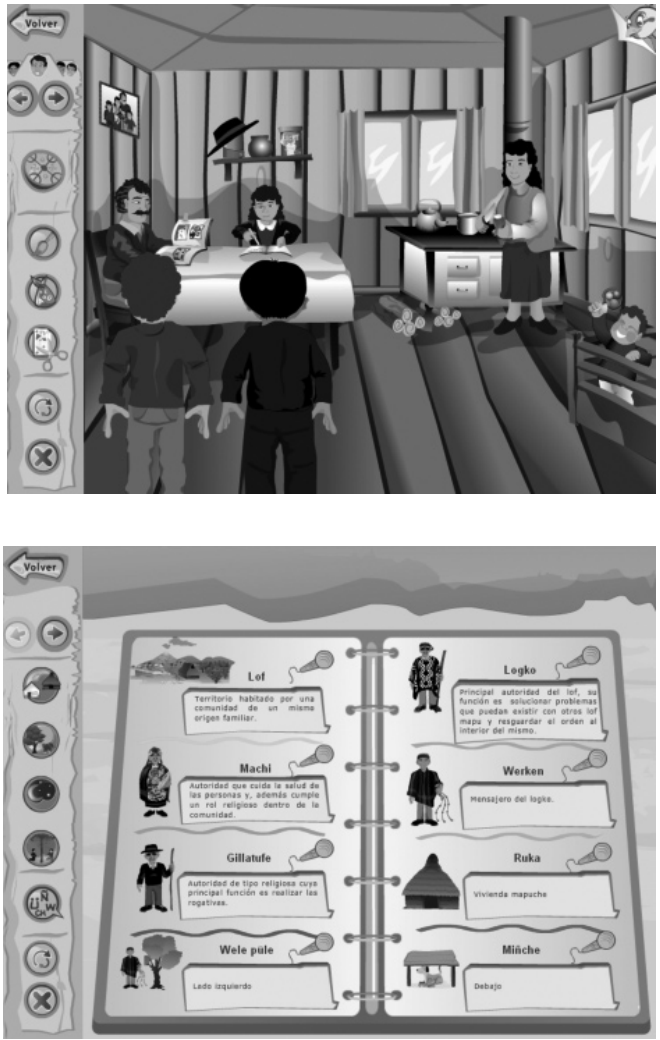

Imágenes del software "Kimkantuain Aprender
Haciendo"

\section{Propuesta del Núcleo Milenio}

En el contexto descrito, se ha reconocido la oportunidad de mejorar diversos aspectos de la educación aprovechando la presencia de tecnologías digitales en las escuelas chilenas en sectores de población vulnerable. Las oportunidades giran en torno a desafíos de equidad, de nivelación de oportunidades entre el mundo rural y el urbano y como medio para el desarrollo de talentos, en especial en contextos mapuche y altamente vulnerables.

\footnotetext{
7 Educarchile: http://www.educarchile.cl/

8 IIE: Instituto de Informática Educativa de la Universidad de La Frontera. http: //www.iie.ufro.cl/
} 
Las tecnologías digitales (computadores, proyectores, Internet, etc.) son una realidad desde hace más de una década en la mayoría de las escuelas chilenas. Cada año, el Ministerio de Educación anuncia más equipamiento y mejor Internet, con lo que estas van adquiriendo año a año más infraestructura digital.

Sin embargo, el uso que se hace de esta infraestructura es aún incipiente. Además, en las escuelas rurales hay menos estudiantes con acceso a computadores en sus hogares e Internet es aún débil. Muchos estudiantes rurales deberán migrar a la ciudad para continuar estudios de enseñanza media y luego estudios superiores, lugares donde el uso de TIC es más intenso, por lo que se encontrarán en desventaja ante sus pares.

En el ámbito del Núcleo Milenio, el objetivo es estructurar una propuesta para el uso de tecnologías digitales en escuelas básicas -urbanas y rurales- con población mapuche. La propuesta incluye elementos metodológicos y contenidos digitales multimediales aprovechando la riqueza de la cultura mapuche.

Se desea apoyar los aprendizajes mínimos insertos en el currículo nacional y desarrollar habilidades en el uso de tecnologías digitales a través de propuestas de trabajo en el aula así como en actividades extra programáticas.

En cuanto a producción de contenidos digitales y equipos, la metodología es el desarrollo incremental de prototipos con evaluación en escuelas adscritas a la Fundación AraucaníAprende ${ }^{9}$ con población escolar mapuche.

Entre los ámbitos de trabajo se encuentran los siguientes:

1. Sistematizar la oferta de tecnología digital educativa en español, en relación a la cultura mapuche, tales como: software educativo, contenidos educativos en Internet, juegos digitales con contenidos interculturales.

2. Diseñar actividades con robots educativos para los niveles pre básico y básico inicial $\left(1^{\circ}\right.$ y $\left.2^{\circ}\right)$ con niños mapuche [9].

3. Desarrollar mecanismos de evaluación del impacto del uso de tecnologías digitales en ambientes educativos interculturales, tales como: impacto en aprendizajes, desarrollo de habilidades TIC, percepción del niño mapuche y su comunidad respecto de la escuela, autoestima, creatividad. 


\section{Referencias}

BARAK, M. (2006). "Instructional principles for fostering learning with ICT: teacher's perspectives as learners and instructors." Education and Information Technologies 11(2): 121-135.

LOVELESS, A. M. (2003). "The Interaction Between Primary Teachers' Perception of ICT and Their Pedagogy". Education and Information Technologies 8(4): 313-326.

PELGRUM, W. J. (2001) ". Obstacles to the integration of ICT in education: results from a worldwide educational assessment". Computers \& Education 37: 163-178.

PELGRUM, W. J. (2002). Teachers, teachers policies and ICT. OECD Seminar: The effectiveness of ICT in schools: Current trends and future prospectus, Tokyo, Japan.

HEPP, P. (2003). Enlaces: el programa de informática educativa de la reforma educacional chilena. Políticas educacionales en el cambio de siglo. La reforma del sistema escolar de Chile. C. Cox. Santiago, Editorial Universitaria.

HEPP, P.; DREVES, C. y LAVAL, E. (2009)."Esfuerzo Público-Privado para avanzar juntos en un desafío central de La Araucanía: Mejoramiento de la Educación en Sectores de Pobreza". Capítulo del libro "Formación Continua de Profesores" Carmen Sotomayor y Horacio Walker (editores). Editorial Universitaria. ISBN 978-956-11-2058-7.

HINOSTROZA, J. E; HEPP, P. y Cox, C. (2009). "National Policies and Practices on ICT in Education. Chile(Enlaces)". Capítulo del libro "Cross-National Information and Communication Technology. Policies and Practices in Education". Tjeerd Plomp, Ronald E. Andersen, Nacy Law y Andreas Quale (editores). $2^{\text {a }}$ edición revisada. Information Age Publishing Inc. ISBN 978-1-60752-043-6.

ZHAO, Y., PUGH, K. et al. (2002). "Conditions for Classroom Technology Innovation". Teachers College Record, Columbia University 104(3): 482-515.
PETRE, M. AND B. PRICE (2004). "Using Robotics to Motivate 'Back Door' Learning". Education and Information Technologies 9(2): 147158.

Lista de algunos sitios Internet internacionales, con contenidos interculturales y uso de tecnologías digitales

UNESCO ICT4ID: ICT for Intercultural Dialogue Developing communication capacities of indigenous peoples: http://portal.unesco.org, http://knet.ca/

NATIVENET: Recursos para culturas indígenas del mundo, http://www.nativeweb.org/ http: / / www.eurosur.org/TIPI/rciidpi.htm

NATIVEWIKI: http://www.nativewiki.org/

CIIDPI Centro Internacional de Información y Documentación de los Pueblos Indígenas www. eurosur.org/TIPI/rciidpi.htm

ONU - G@ID : http://www.un-gaid.org/

INKARRI-NET: Red Telemática de los Pueblos Indígenas http://www.eurosur.org/TIPI/redtelem.htm

CCAB: Canadian Council for Aboriginal Business: http://www.ccab.com/

dgCommunities: Indigenous Issues: http://indigenous.developmentgateway.org/

K-Net: Kuhkenah Network (Primeras Naciones): http://knet.ca/ http://smart.knet.ca/

RICTA: Research on ICT with Aboriginal Communities: http://www.ricta.ca/

IITF: International Indigenous ICT Task Force: http://iictf.blogspot.com/

Community ICT: http://www.communityict. $\mathrm{ca} /$

FIDAMERICA: http: / /www. fidamerica.org/ 